\title{
A few questions regarding perceptual filling-in of the blind spot
}

\author{
Bernt Christian Skottun \\ Ullevaalsalleen $4 \mathrm{C}$ \\ 0852 Oslo \\ Norway \\ e-mail: berntchrskottun@gmail,com
}

Short title: Filling-in

\begin{abstract}
The fact that we are generally unaware of our blind spots is supposed to be the result of the visual system filling them in. This brings up the question of what would be the case if no filling-in were to take place. In other words, what would be the difference between the presence and absence of filling-in. The lack of a clear answer to this question makes it unclear what is to be explained by filling-in or even if any explanation is called for. Because filling-in is supposed to be accomplished by some "mechanism" the lack of an answer raises a question regarding what is to be meant by "mechanism" in this case.
\end{abstract}

Key words: mechanisms; vision; optic disk; blind spot. 
In the retina there is a region, not very far from the fovea, where the optic nerve leaves the eye. In this place-called the the optic disk-there are no photoreceptors. Thus, when viewing the world with one eye there will be a part of the visual field at about 12 - 15 degrees temporally and approximately 1.5 degrees below the horizontal in which we are blind. This area is consequently known as the blind spot. However, we are not usually aware of this blind region. The fact that we are not aware of this has been referred to as a matter of "filling-in", which suggests, or implies, that the visual system in some way fills in the blind area. For instance, Chang and Pearson (2020) listed filling-in as an example of "[t]he constructive nature of vision". As a consequence, explanations of filling-in have been proposed. [FOOTNOTE 1] [FOOTNOTE 2]

However, it is not clear what would be the case if there were no filling-in. In regard to filling-in of the blind spot de Weerd et al. (1998, p 2721) wrote: "The fact that we do not perceive an empty region in our visual field corresponding to the blind spot indicates that the visual system perceptually fills-in the blind spot .,.". However, we are not being told what it would mean "to perceive an empty region". To consider another example, Li et al., (2014, p. 1) wrote "The optic disk of the eye is formed by the ganglion cell axons exiting the eyeball on their way to the brain, resulting in a region of the retina where there are no photoreceptors and thus there should be no vision at all." It is not clear what it would be like for there to be "no vision". That is, it is not clear how "no vision" would differ from the blind spot as we know it. Further on in the same text the authors write in regard to the optic disk "... we never experience a dark hole in our visual field; instead, we perceive a complete visual world." But it is not explained what photoreceptors are supposed to allow us to see the "dark hole". These examples indicate that we lack a coherent account of what vision would be like if no filling-in were to take place. Without such an account we cannot decide what difference filling-in is supposed to make. And without knowing that, how can we know what is to be explained? Or, even that there is something to be explained?

This brings us to the more general issue of "mechanisms". Filling-in has been presented as being the result of the working of some "mechanism" (Baek et al., 2012; Caputo, 1998; Hsu et al., 2006; Komatsu, 2006; Li et al., 2014 Qian et al., 2017; Raman \& Sarkar, 2017; Ramachandran, 1992; Spillmann, 2011; Spillmann et al., 2006). For instance, Spillmann (2011) proposed that filling-in was the result of "cortical mechanisms involving long-range horizontal interactions". However, as we just saw it is far from clear what difference filling-in is supposed to make. Consequently, we are left to ask, what would vision be like without these "long-range cortical mechanisms"? This demonstrates how when in visual science we are faced with some hypothesized mechanism invoked to account for some phenomenon we need to ask: What would be 
the case if this mechanism were to break down? And, if we are unable to answer this question we need to ask: What would be the justification for talking about "a mechanism" in this case?

\section{FOOTNOTE}

1. Pessoa et al., (1998) have indicated that there may be many forms of filling-in. The present analysis deals only with the the kind of filling-in invoked to account for why why we do not see our blind spots.

2. It has been proposed that we do not know what the absence of filling-in is like since it has never happened. This raises the question of how do we know that it has never happened if we do not know what it would be like.

3. Arguments of the present kind also applies to other instances where "mechanisms" have been proposed. For instance, there has been mention of "the mechanisms" for vernier acuity (Levi et al., 2000) and "mechanisms" for feature integrating into coherent percepts (Levi et al., 1997). We are left to ask what would be the case if these mechanisms were to fail? And, if they cannot fail, that is, if they cannot be imagined to fail, what would be the justification for speaking of "mechanisms" in these cases?

\section{REFERENCES}

Baek, Y., Cha, O., Chong, S.C. (2012) Characteristics of the filled-in surface at the blind spot. Vision Research, 58, 33-44.

Caputo, G. (1998) Texture brightness filling-in. Vision Research, 38, 841-851.

Chang, S., \& Pearson, J. (2020) The functional effects of voluntary and involuntary phantom color on conscious awareness. Journal of Experimental Psychology: General, 149, 1006-1016.

de Weerd, P., Desimone, R., \& Ungerleider, L.G. (1998) Perceptual filling-in: a parametric study. Vision Research, 38, 2721-2734.

Komatsu, H. (2006) The neural mechanisms of perceptual filling-in. Nature Reviews Neuroscience, 7, 220231.

Levi, D.M., Klein, S.A. \& Carney, T. (2000) Unmasking the mechanisms for vernier acuity: evidence for a template model of vernier acuity. Vision Research, 40, 951-972.

Levi, D.M., Sharma, V. \& Klein, S.A. (1997) Feature integration in pattern perception. Proceedings of the National Academy of Sciences, 94, 11742-11746.

Li, H., Luo, J., Lu, Y., Kan, J., Spillmann, L., \& Wang, W. (2014) Asymmetrical color filling-in from the nasal to the temporal side of the blind spot. Frontiers in Human Neuroscience, 8, 534.

Pessoa, L. Thompson, E., \& Noe, A. (1998) Finding out about filling-in: A guide to perceptual completion for visual science and the philosophy of perception. Behavioral and Brain Sciences, 21, 723802. 
Qian, C.S., Brascamp, J., \& Liu, T. (2017) On the functional order of binocular rivalry and blind spot filling-in. Vision Research, 136, 15-20.

Ramachandran, V.S. (1992) Blind spots. Scientific American, 266(5), 86-91.

Raman, R., \& Sarkar, S. (2016) Predictive coding: A possible explanation of filling-in at the blind spot. PLoS One. 11(3):e0151194.

Raman, R., \& Sarkar, S. (2017) Significance of natural scene statistics in understanding the anisotropies of perceptual filling-in at the blind spot. Scientific Reports, 7: 3586.

Spillmann, L. (2011) Fading, filling-in, and the perception of uniform surfaces. Chinese Journal of Psychology, 53, 399-411.

Spillmann, L., Otte, T., Hamburger, K., \& Magnussen, S. (2006) Perceptual filling-in from the edge of the blind spot. Vision Research, 46, 4252-4257. 